mometer with a pressure of I atmosphere at about $20^{\circ} \mathrm{K}$. Corrected to the absolute scale the best value would appear to be $45^{\circ} \mathrm{K}$. The triple point, if it exists, is certainly below I $\mathrm{cm}$., perhaps below $7 \mathrm{~mm}$. at which, by corresponding states, the temperature would be about $3^{\circ} \mathrm{K}$., and the liquid remains very mobile.

Liquid helium has a density of $0^{*} \mathrm{I}_{5}$, which gives $b$ a value of $0^{\circ} 00017$, about twice that which has been assumed before from then known properties and used in calculations. From this, again, the critical pressure must be about 2 to 3 atmospheres, so that helium under 5000 would correspond with carbon dioxide under 100,000 atmospheres. At the boiling point the ratio of vapour to liquid density is I : II, which indicates a critical temperature of not much more than $5^{\circ} \mathrm{K}$., and a critical pressure of about $2^{\circ} 3$ atmospheres. Lastly, the value of $a$ will be about 0 00005, the smallest value known, but a most interesting confirmation of van der Waals's contention in 1873 , that there must be some attraction between the molecules of all substances.

Francis Hyndman.

\section{THE ETIOLOGY OF TRYPANOSOMIASIS.}

$\mathrm{I}$ a communication to the Paris Academy of Sciences on February 24, some remarkable discoveries concerning the development of pathogenic trypanosomes in tsetse-flies are brought forward by M., E. Roubaud, member of the Mission Française d'Etudes de la Maladie du Sommeil. Experimenting with four species of pathogenic trypanosomes, namely, Trypanosoma gambiense, $T$. dimorphon, T. brucei, and T. cazalboui, and with Glossina palpalis, Roubaud found that immediately after the fly has fed on the blood of an infected animal, its proboscis contains blood in which the trypanosomes are moving actively. In a very short time, however, the trypanosomes attach themselves to the wall of the proboscis and undergo changes of structure, becoming Herpetomonas-like, with the kinetonucleus in front of the trophonucleus. The undulating membrane has disappeared, and the flagellum, as the organ of fixation, is greatly thickened, so as to resemble a small stalk to the body. These changes are complete in five minutes after ingestion of the blood. The attached parasites at first exhibit active movements of the body. but soon become quiescent; no phenomena of conjugation could be observed, either before or after these changes. But the parasites multiply actively in this situation, forming little tufts or colonies, so that at the end of one hour they have become excessively numerous; they are found attached to the internal face of the labrum, sometimes chiefly at the base of the proboscis, in other cases along its whole length as far as the point. When observed in the salivary fluid they appear immobile, but when treated with serum or with physiological salt-solution they vibrate rapidly and may become free, in which case they swim with the flagellum forward and the hinder part of the body rigid, thus differing greatly in appearance from the original trypanosome-form. The free parasites have a great power of attachment, and when under observation they may fix themselves firmly to the slide.

The author regards this development as a temporary culture or "culture d'attente" of the parasites. Both by observation and experiment he shows that the forms in the proboscis are not derived from trypanosomes regurgitated from the digestive tract of the tsetse. T. brucei was found to die out without multiplication in the intestine of Glossina palpalis in a short time. The culture in the proboscis was found to persist beyond forty-eight hours in the case of $T$. brucei, and for five or six days in the case of the other three species of trypanosomes. Only about ten per cent., however, of the tsetses fed on infected animals developed a culture of the trypanosomes in the pror boscis. On the other hand, the power of multiplying in the proboscis was found to be a specific relation between the trypanosomes and the tsetse.

These observations lack as yet the crucial test of an experimental infection by means of the proboscisculture, but nevertheless they throw great light on the problem of the transmission of pathogenic trypanosomes. It has been shown by previous experimenters that the transmission is effected by the direct or machanical method, and all attempts to prove experimentally an indirect or cyclical mode of transmission have given negative results. That being so, it was difficult to understand why the power of direct transmission should be possessed, apparently, by tsetseflies alone, and not bv other biting insects to an eaual degree. Roubaud's observations show that the pathegenic trypanosomes have a quite specific power of adapting themselves to the salivary secretions of the tsetse, and thus explain the peculiar relation between these flies and the spread of diseases caused by trypanosomes in Africa. Moreover, a very important new line of investigation is indicated by the author's discoveries.

E. A. M.

\section{NOTES.}

Aвout a year ago Sir William Ramsay and Mr. A. T. Cameron announced that they had observed the production of the alkaline metals and lithium in solutions of copper salts submitted to the action of the radium emanation, and concluded that in the presence of the emanation copper underwent a degradation into the elements potassium, sodium, and lithium. In the current number of the Comptes rendus of the Paris Academy of Sciences, Mme. Curie and Mlle. Gleditsch give an account of the attempts they have made to repeat this experiment. They first point out the extreme difficulty of obtaining chemical products free from lithium. This metal was found in distilled water and in nearly all the reagents. If a reagent, free from lithium, is allowed to stand in a glass vessel, traces of this metal are found after some time. Even fused quartz is not a safe material, since both opaque and transparent quartz were found to contain notable amounts, the latter furnishing the larger proportion. The experiments had therefore to be carried out in such a manner that the solutions came in contact with platinum only; the water and the acids necessary for the experiment were re-distilled from platinum and preserved in platinum bottles, and after this treatment no lithium could be detected in the residue from 25 c.c. of the nitric acid, 25 c.c. of hydrofluoric acid, and 250 c.c. of water. The quantities of copper and radium emanation were about the same as those used in the original experiment. The salt residues obtained weighed 0.4 and 0.5 milligram, the control experiments giving 0.3 and 0.2 milligram. Spectroscopic examination of this residue showed it to consist of salts of sodium with a little potassium; the presence of lithium could not be proved. Direct experiments on known mixtures of sodium and lithium sulphates showed that the amount of lithiurn present in the residue, if any, must be less than $0.6 \times 10^{-5}$ milligrams. In conclusion, the authors state that they have been unable to confirm the experiments of Messrs. Ramsay and Cameron. It is impossible to state that no trace of sodium or lithium is formed in this experiment, but they consider that the fact of the formation of these elements cannot be considered as established. 